\title{
Seroprevalence of Fasciola infection among Iranian patients hospitalized during the initial wave of COVID-19
}

\author{
Fatemeh Balazadeh ${ }^{1, B, D, F \oplus}$, Mohammad Zibaei $^{2, A-F \oplus}{ }^{\text {, Aliehsan Heidari }}{ }^{2, C, E-F \oplus}$, Hadis Rastad ${ }^{3, C, F \oplus}$, \\ Farzaneh Firoozeh ${ }^{4, D-F} \oplus$ \\ 1 Student Research Committee, School of Medicine, Alborz University of Medical Sciences, Karaj, Iran \\ ${ }^{2}$ Department of Parasitology and Mycology, School of Medicine, Alborz University of Medical Sciences, Karaj, Iran \\ ${ }^{3}$ Non-communicable Diseases Research Center, Alborz University of Medical Sciences, Karaj, Iran \\ ${ }^{4}$ Department of Microbiology, School of Medicine, Alborz University of Medical Sciences, Karaj, Iran \\ A - Research concept and design, B - Collection and/or assembly of data, C - Data analysis and interpretation, \\ $D$ - Writing the article, E-Critical revision of the article, F- Final approval of the article
}

\begin{abstract}
Balazadeh F, Zibaei M, Heidari A, Rastad H, Firoozeh F. Seroprevalence of Fasciola infection among Iranian patients hospitalized during the initial wave of COVID-19. Med Srod. 2021; 24(1-4): 1-4. doi: 10.26444/ms/141876
\end{abstract}

\section{Abstract}

Introduction. Human fascioliasis is a zoonotic infection caused by the adult stage of the Fasciola species. Fasciola hepatica is a parasite that, following living in its vertebrate host, may reduce the host ss inflammatory immune responses to infectious diseases, including COVID-19. Therefore, because of the completely unproven and limited data on this issue, the study evaluates the prevalence of antibodies against this parasite in patients admitted to hospital due to relatively severe symptoms of COVID-19.

Materials and method. During the first wave of coronavirus-19, investigations were carried out on the serum samples of 182 patients hospitalized with COVID-19, diagnosed by PCR test, and on 210 healthy subjects in Alborz province, Iran, between March - June 2020. The status of anti-Fasciola antibodies was determined in all serum samples by using the ELISA technique. A self-developed questionnaire was used to measure demographic information.

Results. The frequency of Fasciola infection was found to be lesser in the COVID-19 patients than in the healthy control ( $n=4$, $2.2 \%, n=9,4.3 \%$, respectively; $p=0.041$ ). There was no significant difference between COVID-19 patients and the control group in the variables of age, education ( $p>0.05)$.

Conclusions. The study indicates that a significant association exists between Fasciola hepatica seroprevalence and the studied COVID-19 patients and healthy individuals. The results also suggest that fascioliasis may be able to lead to decreased immune responses, thereby reducing the severity and symptoms of COVID-19 disease.

\section{Key words}

seroepidemiology, fascioliasis, Fasciola hepatica, coronavirus disease-19, COVID-19, ELISA

\section{INTRODUCTION}

Fascioliasis is a widespread, zoonotic parasitic disease caused by the adult stages of either Fasciola hepatica and / or Fasciola gigantica [1]. Fasciola infection is common in many parts of the world, especially in areas where livestock is more prevalent $[2,3,4]$. Fascioliasis is a vector-borne parasitic disease presenting the widest latitudinal, longitudinal and altitudinal distribution known at present, and infecting herbivorous mammals and humans worldwide $[3,5]$. Fasciola infection appears mainly in Europe, the Americas and Oceania, and some countries in Africa and Asia. According to the WHO, Iran is an important endemic area of fascioliasis, having many health problems and economic losses among the human and animal populations $[6,7]$.

Diagnosis of human fascioliasis is challenging and the performance of parasitological approaches - microscopybased detection of parasite's egg in the stool - are not satisfactory. Therefore, serological methods for the diagnosis

Address for correspondence: Mohammad Zibaei, Department of Parasitology and Mycology, School of Medicine, Alborz University of Medical Sciences, Talaghani Square, North Talaghani Blvd, 3149779453, Karaj, Iran

E-mail: zibaeim@sums.ac.ir of acute-phase and ectopic fascioliasis are mainly based on the detection of anti-Fasciola antibodies in serum. $[8,9,10]$. It has been shown that the parasites can suppress host immune responses. They suppress some of the pathways involved in activating immune responses, such as the presentation of antigens by dendritic cells, the production of cytokines by $\mathrm{T}$ cells (T-lymphocytes), the production of antibodies by $\mathrm{B}$ cells (B-lymphocytes), and the release of alarming by epithelial cells [11]. The also modulate other pathways, such as differentiation of Th cells (T-helper lymphocytes) subsets and B cells isotype changes, as well as inducing other pathways such as Treg cells (Regulatory T-lymphocytes) and Breg cells (Regulatory B-lymphocytes) differentiation and tolerant responses of dendritic cells. Further details are under investigation. As more information becomes available on how anti-parasitic responses are produced, scientists are also discovering new ways to modulate the immune system by these parasites, and use parasitic molecules as treatments for inflammatory diseases [11].

Coronaviruses are in the Orthocoronavirinae subfamily, Coronaviridae family, and Nidovirales order. In the Orthocoronavirinae subfamily, there are four species of alpha $(\alpha)$, beta $(ß)$, gamma $(\gamma)$, and delta $(\delta)$ coronavirus. Alpha 
and beta types are pathogenic in mammals while gamma and delta types in birds [12]. The coronavirus disease-19, known as COVID-19, which was first identified in Wuhan, China, and quickly spread to other countries and caused the recent pandemic that led to the infection and death of many people worldwide [12]. A report indicates that out of 425 patients studied in Wuhan, about $55 \%$ had a history of employment in the seafood wholesale market. Therefore, it has been shown that the disease can be transmitted from animals to humans [13].

\section{OBJECTIVE}

Due to the importance of this helminthic zoonosis infection and the lack of data about its prevalence in Iran, the high rate of its migration, the report of COVID-19 patients, prevalence of definitive hosts in the region, the study of new foci of fascioliasis became the area of the presented study $[14,15]$. The aim was to investigate fascioliasis among the COVID-19 patients, and the epidemiological factors associated with the infectio, and compare them with a healthy population in Iran.

\section{MATERIALS AND METHOD}

Ethical consideration. Informed consent was obtained from all subjects, and all methods were carried out in accordance with the relevant guidelines and regulations of the Ethics Committee of the Alborz University of Medical Sciences (IR. ABZUMS.REC.1399.215). Written informed consent was also obtained from the guardians of patients who were minors or children, and illiterate patients enrolled in the study.

Study area and subjects. Karaj, the capital of Alborz province, is located at latitude $35^{\circ} 49^{\prime}$ north and longitude $50^{\circ} 59^{\prime}$ east, on the low, flat plain of the Karaj River in the central part of the country (Fig. 1), a sub-tropical area with long hours of sunshine, high temperature and humidity. Karaj has a total area of $162.14 \mathrm{~km}^{2}$ with a population of approximately two million. The studied sites covered an area of $115.3 \mathrm{~km}^{2}$ with 1,270,000 inhabitants. (https://en.wikipedia.org/wiki/ Alborz_Province).

The cross-sectional study was conducted during the initial wave of coronavirus disease-19 on 182 hospitalized patients (86 women and 96 men) with COVID-19, aged between 14-72 years (mean 55.17, \pm 19.04 ) who were followed-up in the Infectious Diseases Division of Shahid Rejaei Hospital (as a referral centre) between March - June 2020. The clinical history of each patent was evaluated, as well as the results of Computed Tomography (CT) scans or serological examination. The diseases were defined accordingly to the Polymerase Chain Reaction (PCR). The control group consisted of 210 people: 123 women and 87 men, volunteers aged between 10-69 years (mean 30.29, \pm 12.14 ) with comparable epidemiological characteristic and without any complaints or history of previous coronaviruses diseases 2019 in either themselves or their families.

Serological technique. Three $\mathrm{mL}$ venous blood was taken under sterile conditions from each individual in both groups and the sera separated by centrifuge at 2,500 rpm, aliquot, and stored at $-20^{\circ} \mathrm{C}$ until analyses were carried out. AntiFasciola antibodies were detected by commercial ELISA kit (Pishtaz Teb Medical Research Company, Tehran, Iran), following the manufacture's instruction. Briefly, ELISA microplates were coated with Fasciola hepatica antigens, the serum samples were added to the plates and incubated at room temperature for one hour. The plates were washed again, and diluted anti-human IgG horseradish peroxidase conjugated was added to each well and incubated for one hour at $37^{\circ} \mathrm{C}$. After washing three times, the plates were incubated with chromogen/substrate and were stopped bythe addition

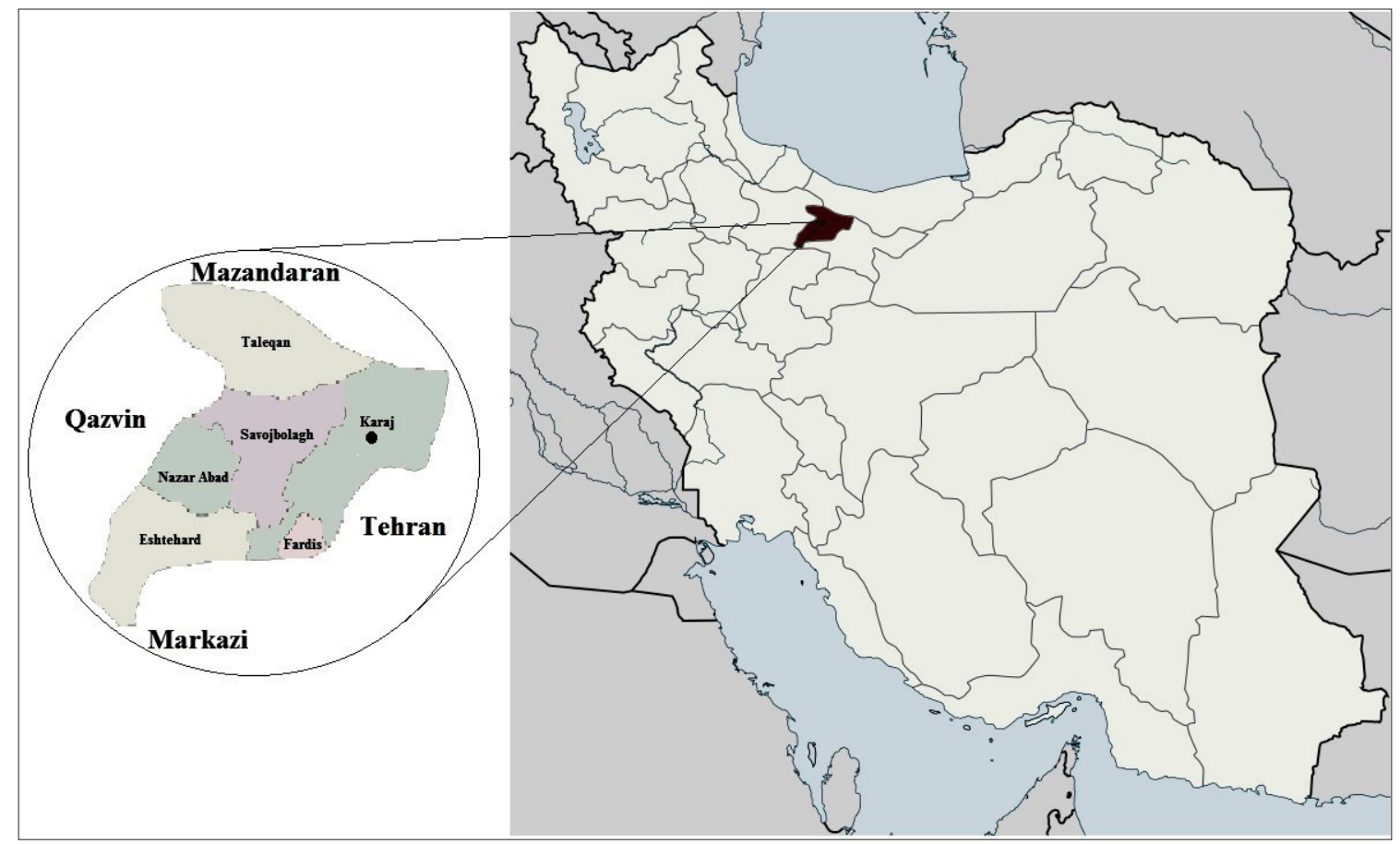

Figure 1. Schematic map of the study areas, Alborz province, Iran 
of a stopper solution. The optical density (OD) of samples was monitored at a wavelength of $450 \mathrm{~nm}$ using a microplate reader (STAT-FAX-2100-OHAHIO-USA).

Statistical analysis. The resulting data were analyzed by T-test, non-parametric, and Mann-Whitney $U$ tests. Associations were statistically significant when a $p$-value of less than 0.05 was observed.

\section{RESULTS}

Out of 182 COVID-19 hospitalized patients who participated in the study, 4 (2.2\%) were positive for anti-Fasciola hepatica antibodies, compared to nine (4.3\%) among the healthy group. This was statistically significant $(p=0.041)($ Tab. 1$)$. There was no significant difference between COVID-19 patients and the healthy control participants in terms of education levels, age groups, and gender. Table 2 presents details of the Fasciola seroprevalence, both in all the studied people as well as according to occupation. In COVID-19 patients, the seropositivity rate was significantly higher in housekeepers $(\mathrm{n}=3,75.0 \%)$ among the seropositive subjects, than among workers $(n=1,25.0 \%)(p=0.045)$. A significant association was found between the prevalence of fascioliasis with the occupation variable in the studied subjects $(p=0.038)$.

The seroprevalence rate in the COVID-19 hospitalized patients was substantially higher among the people aged $40-59$ years $(n=2,50.0 \%)$ than in older subjects $(60-79$ years, $\mathrm{n}=1,25.0 \%$; and $\geq 80$ years, $\mathrm{n}=1,25.0 \%)(\mathrm{p}=0.067)$. High-titer antibodies were most prevalent among those in the age group 40-59 years, suggesting that recent or current exposure to Fasciola is most common in this age group (Tab. 2).

Seropositivity was more prevalent in females $(n=3,75.0 \%)$ than in males $(n=1,25.0 \%)$ related to the COVID-19 and showed statistical significance $(p=0.032)$. Table 2 shows that no association was found between the gender variable in the positive cases of fascioliasis in the studied participants $(\mathrm{p}=0.262)$.

Based on the results of T-test, no significant difference was found between the seroprevalence of fascioliasis and the studied groups due to educational levels $(\mathrm{p}=0.071)$. Therefore, the seroprevalence of Fasciola infection in the COVID-19 patients with some high school $(n=2)$, high school $(n=1)$, and graduate school $(n=1)$ was not significant.

There was a predominance of rural compared to urban population among the seropositive COVID-19 patients. Of the four (2.2\%) seropositive hospitalized patients, there were three (75.0\%) from a rural area, and one (25.0\%) from an urban are, which was statistically significant $(\mathrm{p}=0.001)$. According to the place residence, the seroprevalence of Fasciola infection was higher among participants from control group than in those from COVID-19 patients. This difference was statistically significant $(p=0.004)$.

\section{DISCUSSION}

Parasitic infections, especially helminthic diseases, adapt to their host during long-term infections which usually lead to chronic disease with reduced mortality. During this evolutionary adaptation to their hosts, including vertebrates, parasites have helped modulate several molecular and physiological mechanisms, for example, the host immune system. Thus, the parasitic helminths elicit modulated Th2
Table 1. Seroprevalence of Fasciola infection antibodies in COVID-19 ${ }^{a}$ patients and control group

\begin{tabular}{lcccccc}
\hline & \multicolumn{2}{c}{ COVID-19 patients } & \multicolumn{2}{c}{ Control group } & Total & P-value \\
\cline { 2 - 7 } & $\mathrm{N}$ & $\%$ & $\mathrm{~N}$ & $\%$ & & \\
\hline Seropositive & 4 & 2.20 & 9 & 4.30 & 14 & 0.041 \\
\hline Seronegative & 178 & 97.80 & 201 & 95.70 & 378 & \\
\hline Total & 182 & 100 & 210 & 100 & 392 & \\
\hline
\end{tabular}

${ }^{a}$ Coronavirus disease-19

Table 2. Analysis of factors significantly associated with Fasciola infection in COVID-19 patients and the healthy control group

\begin{tabular}{lcccccc}
\hline \multirow{2}{*}{ Variables } & \multicolumn{2}{c}{ COVID-19 patients } & \multicolumn{2}{c}{ Control group } & \multirow{2}{*}{ Total $\begin{array}{l}\text { Statistical } \\
\text { (P-value) }\end{array}$} \\
\cline { 2 - 5 } & $\mathrm{N}$ & $\%$ & $\mathrm{~N}$ & $\%$ & & \\
\hline Age Categories (years) & & & & & & \\
\hline$\geq 19$ & 2 & 1.1 & 1 & 0.5 & 3 & 0.578 \\
\hline $20-39$ & 39 & 21.4 & 20 & 9.5 & 59 & \\
\hline $40-59$ & 56 & 30.8 & 75 & 35.7 & 131 & \\
\hline $60-79$ & 66 & 36.3 & 87 & 41.4 & 153 & \\
\hline $80 \leq$ & 19 & 10.4 & 27 & 12.9 & 46 & \\
\hline Gender & & & & & & \\
\hline Female & 86 & 47.3 & 123 & 58.6 & 209 & 0.262 \\
\hline Male & 96 & 52.7 & 87 & 41.4 & 183 & \\
\hline
\end{tabular}

Education

\begin{tabular}{lcccccc}
\hline No School & 7 & 3.9 & 3 & 1.4 & 10 & 0.071 \\
\hline Some high School & 39 & 21.4 & 36 & 17.1 & 75 & \\
\hline High School & 79 & 43.4 & 104 & 49.6 & 183 & \\
\hline $\begin{array}{l}\text { Some college/ } \\
\text { Graduate school }\end{array}$ & 57 & 31.3 & 67 & 31.9 & 124 & \\
\hline
\end{tabular}

Graduate schoo

Occupation

\begin{tabular}{lcccccc}
\hline Unemployed & 100 & 54.9 & 39 & 18.6 & 139 & 0.038 \\
\hline Worker & 15 & 8.2 & 51 & 24.3 & 66 & \\
\hline Housekeeper & 34 & 18.8 & 45 & 21.4 & 79 & \\
\hline Farmer & 8 & 4.4 & 15 & 7.2 & 23 & \\
\hline Student & 15 & 8.2 & 36 & 17.1 & 51 & \\
\hline Other & 10 & 5.5 & 24 & 11.4 & 34 & \\
\hline Residency & & & & & & \\
\hline Rural & 110 & 60.4 & 174 & 82.9 & 284 & 0.004 \\
\hline Urban & 72 & 39.6 & 36 & 17.1 & 108 & \\
\hline
\end{tabular}

responses in their vertebrate hosts, resulting in immune response with a well-controlled inflammatory component, including inhibition of pro-inflammatory cytokines and induction of hyporesponsive state by IL-10 (Interleukin-10) and produces Treg cells [11]. In addition, the health hypothesis suggests that the absence of helminthic infections in the population of developed countries and the absence of parasitic immune stimulants increase autoimmune diseases with an exacerbated inflammatory komponent, such as asthma and allergies. On the other hand, allergic diseases are increasing at an alarming rate worldwide, particularly in developed countries.

In contrast, there is a decrease in the prevalence of helminthic infections and other neglected diseases [16]. Therefore, it can be concluded that infection with Fasciola hepatica, as a zoonotic helminthic infection, from which 
various immunomodulators molecules have been isolated, can lead to a milder course of COVID-19 disease [17]. It is speculated that Fasciola hepatica, can lead to decreased immune responses, thereby reducing the severity and symptoms of COVID-19 disease and, consequently, the hospitalization of COVID-19 patients. Therefore, it can be hypothesized that the prevalence of fascioliasis in the COVID-19 patients who had admitted to hospital is lower than the normal population.

In the current study, the frequency of Fasciola hepatica infection in the COVID-19 patients was $2.2 \%$, lesser than the healthy control without COVID-19 symptoms (4.3\%). However, as the reported prevalence of fascioliasis in the general population in Iran is up to $50.0 \%$, the relatively high frequency of Fasciola infection in the control group requires cautious interpretation.

Of the 4 COVID-19 patients seropositive to Fasciola, three (75.0\%) were female; however, this was higher than the overall proportion of female patients (47.3\%). Fascioliasis is seen more frequently among middle-aged adults, probably due to exposure to the infectious agent. The relatively gradual increase in seroprevalence associated with age suggested that the ingestion of metacercariae in water or leafy vegetables that grow near water, which is greatest during adulthood, may not be the principal mechanism by which people are exposed to Fasciola hepatica. Therefore, given that the disease is transmitted through consumption of contaminated water or food, the infection can be easily controlled and prevented by avoiding contaminated water and food [18].

In this study, COVID-19 hospitalized patients consisted of various age groups from young adults to the elderly with ages ranging between $14-72$ years. The seropositivity rate was not affected by age, although there were significantly more seropositive COVID-19 patients who were housekeepers.

A main finding of the study was the significant different between seroprevalence in the rural population compared with the urban population to Fasciola. Of the four patients classified as having anti-Fasciola antibodies, $75.0 \%$ resided in a rural area. These observations suggest that the population in rural areas were more likely to be exposed to the parasite.

This study has some limitations. First, this study is a casecontrol study at a particular time, so the causal relationship between the research variables cannot be fully inferred. Secondly, because of the limited studies of Fasciola infection in patients with COVID-19 disease, the results of this study cannot be considered consistent or inconsistent with other studies.

\section{CONCLUSION}

To conclude, fascioliasis is a helminthic disease which is a preventable and treatable disease. The findings of the present study further corroborate some observation of an association between Fasciola infection and COVID-19 disease. Therefore, it is suggested that further studies should be conducted to obtain more reliable results regarding the effects of this parasites infection on the human immune system, and their effect on the onset and symptoms of other diseases, such as COVID-19, which have not yet been effective.

\section{REFERENCES}

1. Molyneux DH, Savioli, Engels D. Neglected tropical diseases: progress towards addressing the chronic pandemic. Lancet. 2017; 389(10066): 312-325. http://doi.org/10.1016/S0140-6736(16)30171-4

2. Doyle ME. Foodborne parasites a review of the scientific literature. Food Research Institute University of Wisconsin-Madison, Madison WI 53706, 2003, 281-394.

3. Mas-Coma S, Valero MA, Bargues MD. Fascioliasis. Adv Exp Med Biol. 2019; 1154: 71-103. http://doi.org/10.1007/978-3-030-18616-6_4 4.Dietrich CF, Kabaalioglu A, Brunetti E, Richter J. Fasciolosis. Z Gastroenterol. 2015; 53(4): 285-290. http://doi. org/10.1055/s-0034-1385728

5. Muñoz Zambrano ME, Placencia Medina M, Del Pozo Muñoz JA, Sevilla Andrade C, Huiza Franco A. Serological diagnosis of Fasciola hepatica infection: a systematic review. Rev Gastroenterol Peru. 2020; 40(2): 155-161.

6. Mitra AK, Mawson AR. Neglected tropical diseases: epidemiology and global burden. Trop Med Infect Dis. 2017; 2(3): 36. http://doi. org/10.3390/tropicalmed2030036.

7.Heydarian P, Ashrafi K, Rahimi Esboei B, Mohebali M, Kia EB, Aryaeipour M, et al. Emerging cases of fascioliasis in Lorestan Province, Western Iran: case Series report. Iran J Public Health. 2021; 50(1): 195-200. http://doi.org/10.18502/ijph.v50i1.5087.

8. Sarkari B, Khabisi SA. Immunodiagnosis of human fascioliasis: an update of concepts and performances of the serological assays. J Clin Diagn Res. 2017; 11(6): OE05-OE10. http://doi.org/10.7860/ JCDR/2017/26066.10086.

9. Shafiei R, Sarkari B, Sadjjadi SM. Performance of a $27 \mathrm{kDa}$ Fasciola hepatica antigen in the diagnosis of human fascioliasis. J Lab Physicians. 2015; 7(1): 17-20. http://doi.org/10.4103/0974-2727.154781.

10. Ashrafi K. The status of human and animal fascioliasis in Iran: A narrative review article. Iran J Parasitol. 2015; 10(3): 306-328.

11. Maizels RM, McSorley HJ. Regulation of the host immune system by helminth parasites. J Allergy Clin Immunol. 2016; 138(3): 666-675. http://doi.org/10.1016/j.jaci.2016.07.007.

12. Li H, Liu SM, Yu XH, Tang SL, Tang CK. Coronavirus disease 2019 (COVID-19): current status and future perspectives. Int J Antimicrob Agents. 2020; 55(5): 105951. http://doi.org/10.1016/j. ijantimicag.2020.105951.

13. Ahmad T, Khan M, Haroon, Musa TH, Nasir S, Hui J, et al. COVID-19: Zoonotic aspects. Travel Med Infect Dis. 2020; 36: 101607. http://doi. org/10.1016/j.tmaid.2020.101607.

14. Hatami H, Asmar M, Masoud J, Mansouri F, Namdaritabar H, Ramazankhani A. The First epidemic and new-emerging human fascioliasis in Kermanshah (western Iran) and a ten-year follow up, 1998-2008. Int J Prev Med. 2012; 3(4): 266-272.

15. Moshfe A, Aria A, Erfani N, Jamshidi A, Sarkari B, Abdolahi Khabisi $S$, et al. Clinical features, diagnosis and management of patients with suspicion of fascioliasis in Kohgiluyeh and Boyer-Ahmad province, southwestern Iran. Iran J Parasitol. 2020; 15(1): 84-90.

16. Frigerio S, da Costa V, Costa M, Festari MF, Landeira M, RodríguezZraquia SA, et al. Eosinophils control liver damage by modulating immune responses against Fasciola hepatica. Front Immunol. 2020; 11: 579801. http://doi.org/10.3389/fimmu.2020.579801.

17. Siles-Lucas M, González-Miguel J, Geller R, Sanjuan R, Pérez-Arévalo J, Martínez-Moreno Á. Potential influence of helminth molecules on COVID-19 Pathology. Trends Parasitol. 2021; 37(1): 11-14. http://doi. org/10.1016/j.pt.2020.10.002.

18. Kumar P, Sunita K, Singh RN, Singh DK. Fascioliasis: a fluke infection is food-borne parasitic diseases and controls their vectors. Int J Biol Med Res. 2020; 11(1): 6982-6989. 